Table S1.A.1 PCR conditions used for mitochondrial genes

\begin{tabular}{|c|c|c|c|c|c|c|}
\hline \multirow[t]{23}{*}{ Thaumetopoea bonjeani } & FW primer & RV primer & MIX & $\operatorname{AT}\left({ }^{\circ} \mathrm{C}\right)$ & EXT & $n^{\circ}$ cycles \\
\hline & TFJ6400 & MPE_a_nad5 & 1 & 55 & 1'30" & 35 \\
\hline & allnd5form & MPE_a_nad5 & 1 & 50 & $1 ' 45^{\prime \prime}$ & 35 \\
\hline & nad5pbihF & nad5pbihR & 1 & 55 & $1^{\prime}$ & 35 \\
\hline & N5J7572 & N4N8727 & 1 & 53 & $1 ' 45^{\prime \prime}$ & 35 \\
\hline & pf7925 & pr8776 & 1 & 55 & 1'30" & 35 \\
\hline & CBJ10933 & CBN11367 & 1 & 58 & $1^{\prime}$ & 35 \\
\hline & CBJ10933 & TS1N11683 & 1 & 52 & $1^{\prime}$ & 35 \\
\hline & CBJ11485 & N1N12442 & 1 & 54 & 1'30" & 35 \\
\hline & N1J12261m & LRN13000 & 2 & 52 & $1^{\prime}$ & 35 \\
\hline & LRJ12887 & LRN13398 & 1 & 58 & $1^{\prime}$ & 35 \\
\hline & eochl10for & OC10rr & 2 & 48 & 1'30" & 35 \\
\hline & SRJ14197 & SRN14745 & 1 & 54 & 1'30" & 35 \\
\hline & SRJ14233 & SRN14588 & 1 & 60 & $30 "$ & 35 \\
\hline & pB12Sfor & pB12Srev & 1 & 53 & $1^{\prime}$ & 35 \\
\hline & LCO1490 & HCO2198 & 1 & 50 & $1^{\prime}$ & 35 \\
\hline & C1J1718 & C1N2353 & 1 & 50 & $1^{\prime}$ & 35 \\
\hline & C1J1751 & TL2N3014 & 1 & 48 & $2^{\prime}$ & 35 \\
\hline & allC1J1922 & allC1N3120 & 1 & 52 & 1'30" & 35 \\
\hline & C1J2441 & C2N3661 & 1 & 52 & 1'30" & 35 \\
\hline & C1J2792 & TKN3796 & 1 & 49 & 1'30" & 40 \\
\hline & C2for2 & A6N4552m & 2 & 57 & 1'30" & 35 \\
\hline & C2J3624 & C3N4908 & 1 & 52 & 1'30" & 35 \\
\hline \multirow[t]{18}{*}{ Thaumetopoea herculeana } & FW primer & RV primer & MIX & $\operatorname{AT}\left({ }^{\circ} \mathbf{C}\right)$ & EXT & $n^{\circ}$ cycles \\
\hline & TFJ6400 & MPE_a_nad5 & 1 & 55 & 1'30" & 35 \\
\hline & N5J7572 & N4N8727 & 1 & 53 & 1'45" & 35 \\
\hline & nad5hwF & nad5hwR & 1 & 55 & $1^{\prime}$ & 35 \\
\hline & pf7925 & pr8776 & 1 & 55 & 1'30" & 35 \\
\hline & CBJ10933 & CBN11367 & 1 & 58 & $1^{\prime}$ & 35 \\
\hline & ochlnad1for & allnd1rev & 1 & 57 & 1'40" & 35 \\
\hline & CBJ11335 & allnd1rev & 1 & 57 & $1^{\prime} 40^{\prime \prime}$ & 35 \\
\hline & CBJ11545 & LRN12866 & 1 & 57 & 1'30" & 35 \\
\hline & OC7rr & allnd1rev & 1 & 54 & 1'30" & 35 \\
\hline & pth1612SAf & pth1612SAr & 1 & 58 & $1^{\prime} 45^{\prime \prime}$ & 35 \\
\hline & N1J12261m & 071105rev5 & 1 & 55 & $1^{\prime}$ & 35 \\
\hline & pth1612SBf & pth1612SBr & 1 & 58 & $1^{\prime} 45^{\prime \prime}$ & 35 \\
\hline & SRJ14197 & TMN200 & 1 & 61 & $2^{\prime}$ & 35 \\
\hline & SRJ14233 & SRN14588 & 1 & 52 & $1^{\prime}$ & 30 \\
\hline & LCO1490 & HCO2198 & 1 & 50 & $1^{\prime}$ & 35 \\
\hline & allC1J1922 & allC1N3120 & 1 & 52 & 1'30" & 35 \\
\hline & C1J2441 & TKN3785 & 1 & 50 & $2^{\prime}$ & 35 \\
\hline
\end{tabular}


Table S1.A.2 PCR conditions used for mitochondrial genes

\begin{tabular}{|c|c|c|c|c|c|c|}
\hline \multirow[t]{4}{*}{ Thaumetopoea herculeana } & FW primer & RV primer & MIX & $\mathrm{AT}\left({ }^{\circ} \mathrm{C}\right)$ & EXT & $\mathrm{n}^{\circ}$ cycles \\
\hline & C2J3399 & A8N4061 & 1 & 50 & $1^{\prime}$ & 35 \\
\hline & TKJ3790 & C3N4908 & 1 & 53 & $1^{\prime} 45^{\prime \prime}$ & 35 \\
\hline & allco1for & allco3rev & 1 & 61 & 2'30" & 40 \\
\hline \multirow[t]{27}{*}{ Thaumetopoea ispartaensis } & FW primer & RV primer & MIX & $\mathrm{AT}\left({ }^{\circ} \mathrm{C}\right)$ & EXT & $n^{\circ}$ cycles \\
\hline & TFJ6400 & MPE_a_nad5 & 1 & 55 & 1'30" & 35 \\
\hline & TFJ6400 & N5N7793 & 1 & 54 & $1^{\prime} 45^{\prime \prime}$ & 35 \\
\hline & allnd5form & MPE_a_nad5 & 1 & 50 & $1^{\prime} 45^{\prime \prime}$ & 35 \\
\hline & N5J7572 & N4N8727 & 1 & 53 & 1'45" & 35 \\
\hline & tisp0710nd5f & tisp0710nd5r & 1 & 52 & $45^{\prime \prime}$ & 35 \\
\hline & nad5pbihF & nad5pbihR & 1 & 55 & $1^{\prime}$ & 35 \\
\hline & pf7925 & pr8776 & 1 & 55 & 1'30" & 35 \\
\hline & CBJ10933 & N1N12595 & 1 & 52 & 1'45" & 40 \\
\hline & CBJ10933 & allnd1rev & 2 & 57 & 1'30" & 35 \\
\hline & N1J12261m & LRN13000 & 2 & 52 & $1^{\prime}$ & 35 \\
\hline & LRJ12888 & LRN13398 & 1 & 57 & $1^{\prime}$ & 35 \\
\hline & ochl16S12Sfor & OC10rr & 1 & 62 & 1'15" & 35 \\
\hline & LRJ12888 & OC10rr & 1 & 61 & 1'30" & 35 \\
\hline & 071105for 1 & 071105rev2 & 1 & 55 & 1'30" & 35 \\
\hline & LRJ13900 & SRN14745 & 1 & 57 & 1'30" & 35 \\
\hline & pB12Sfor & pB12Srev & 1 & 53 & $1^{\prime}$ & 35 \\
\hline & LCO1490 & HCO2198 & 1 & 50 & $1^{\prime}$ & 35 \\
\hline & C1J1718 & C1N2353 & 1 & 50 & $1^{\prime}$ & 35 \\
\hline & C1J1718 & C1N2776 & 1 & 52 & $1^{\prime}$ & 35 \\
\hline & allC1J1922 & allC1N3120 & 1 & 52 & 1'30" & 35 \\
\hline & C1J2441 & TKN3785 & 1 & 50 & $2^{\prime}$ & 35 \\
\hline & C1J2441 & C2N3661 & 1 & 52 & 1'30" & 35 \\
\hline & C2J3399 & A8N4061 & 1 & 48 & $1^{\prime}$ & 35 \\
\hline & C2J3624 & C3N4908 & 1 & 52 & $2^{\prime}$ & 35 \\
\hline & eochl14for & eochl14rev & 1 & 54 & $2^{\prime}$ & 35 \\
\hline & TKJ3790 & C3N4908 & 1 & 62 & $2^{\prime}$ & 35 \\
\hline \multirow[t]{9}{*}{ Thaumetopoea libanotica } & FW primer & RV primer & MIX & $\mathrm{AT}\left({ }^{\circ} \mathrm{C}\right)$ & EXT & $n^{\circ}$ cycles \\
\hline & TFJ6400 & N5N7211m & 1 & 55 & $1^{\prime} 45^{\prime \prime}$ & 35 \\
\hline & allnd5form & MPE_a_nad5 & 1 & 50 & 1'45" & 35 \\
\hline & pf7925 & pr8776 & 1 & 55 & 1'30" & 35 \\
\hline & N5J7572 & N4N8727 & 1 & 53 & $1^{\prime} 45^{\prime \prime}$ & 35 \\
\hline & N5J7806 & N4N8727 & 1 & 57 & $1^{\prime} 45^{\prime \prime}$ & 35 \\
\hline & CBJ10933 & allnd1rev & 2 & 57 & 1'30" & 35 \\
\hline & CBJ10933 & allcobrev & 1 & 55 & $45^{\prime \prime}$ & 35 \\
\hline & CBJ10933 & N1N12595 & 1 & 52 & $1^{\prime} 45^{\prime \prime}$ & 40 \\
\hline
\end{tabular}


Table S1.A.3 PCR conditions used for mitochondrial genes

\begin{tabular}{|c|c|c|c|c|c|c|}
\hline \multirow[t]{18}{*}{ Thaumetopoea libanotica } & FW primer & RV primer & MIX & $\operatorname{AT}\left({ }^{\circ} \mathbf{C}\right)$ & EXT & $n^{\circ}$ cycles \\
\hline & CBJ11335 & N1N12442 & 1 & 54 & 1'30" & 35 \\
\hline & nd1micro & eochl7rev & 1 & 58 & $45^{\prime \prime}$ & 35 \\
\hline & N1J12261m & LRN13000 & 2 & 52 & $1^{\prime}$ & 35 \\
\hline & LRJ12888 & LRN13398 & 1 & 57 & $1^{\prime}$ & 35 \\
\hline & LRJ12888 & OC10rr & 1 & 61 & 1'30" & 35 \\
\hline & ochl16S12Sfor & OC10rr & 1 & 62 & 1'15" & 35 \\
\hline & SRJ14197 & SRN14745 & 1 & 54 & 1'30" & 35 \\
\hline & pB12Sfor & pB12Srev & 1 & 53 & $1^{\prime}$ & 35 \\
\hline & 071105for 1 & 071105rev2 & 1 & 55 & 1'30" & 35 \\
\hline & LCO1490 & HCO2198 & 1 & 50 & $1^{\prime}$ & 35 \\
\hline & LCO1490 & C1N2353 & 1 & 56 & $1^{\prime}$ & 35 \\
\hline & allC1J1922 & allC1N3120 & 1 & 52 & 1'30" & 35 \\
\hline & C1J2183 & C2N3661 & 1 & 48 & $2^{\prime}$ & 35 \\
\hline & C1J2441 & C2N3661 & 1 & 52 & 1'30" & 35 \\
\hline & C1J2441 & TKN3785 & 1 & 50 & $2^{\prime}$ & 35 \\
\hline & allco1for & allco3rev & 1 & 61 & 2'30" & 40 \\
\hline & TKJ3790 & C3N4908 & 1 & 62 & $2^{\prime}$ & 35 \\
\hline \multirow[t]{23}{*}{ Thaumetopoea pinivora } & FW primer & RV primer & MIX & $\mathrm{AT}\left({ }^{\circ} \mathrm{C}\right)$ & EXT & $n^{\circ}$ cycles \\
\hline & PRND51r & N4N8727 & 1 & 54 & 2'30" & 35 \\
\hline & allnd5form & MPE_a_nad5 & 1 & 50 & 1'45" & 35 \\
\hline & nad5pbihF & nad5pbihR & 1 & 55 & $1^{\prime}$ & 35 \\
\hline & N5J7572 & N4N8727 & 1 & 53 & $1^{\prime} 45^{\prime \prime}$ & 35 \\
\hline & pf7925 & pr8776 & 1 & 55 & 1'30" & 35 \\
\hline & CBJ10933 & CBN11367 & 1 & 58 & $1^{\prime}$ & 35 \\
\hline & nd1micro & 12Srev2 & 1 & 50 & $1^{\prime}$ & 35 \\
\hline & OC7rr & allnd1rev & 1 & 54 & 1'30" & 35 \\
\hline & tpin0710cobf & tpin0710cobr & 1 & 52 & $45^{\prime \prime}$ & 35 \\
\hline & CBJ11335 & N1N12442 & 1 & 54 & 1'30" & 35 \\
\hline & CBJ11545 & LRN12866 & 1 & 57 & 1'30" & 35 \\
\hline & LRJ12887 & LRN13398 & 1 & 58 & $1^{\prime}$ & 35 \\
\hline & LRJ12888 & OC10rr & 1 & 61 & 1'30" & 35 \\
\hline & 16Sfor2 & 12Srev2 & 1 & 50 & $1^{\prime} 30^{\prime \prime}$ & 35 \\
\hline & N1J12261m & LRN13000 & 2 & 52 & $1^{\prime}$ & 35 \\
\hline & ochl16S12Sfor & SRN14220 & 1 & 61 & 1'30" & 35 \\
\hline & SRJ14233 & SRN14588 & 1 & 52 & $1^{\prime}$ & 30 \\
\hline & LRJ13342 & SRN14745 & 2 & 52 & $2^{\prime}$ & 35 \\
\hline & SRJ14197 & TMN200 & 1 & 61 & $2^{\prime}$ & 35 \\
\hline & LCO1490 & HCO2198 & 1 & 50 & $1^{\prime}$ & 35 \\
\hline & C1J1718 & TL2N3014 & 1 & 48 & 1'30" & 35 \\
\hline & allC1J1922 & allC1N3120 & 1 & 52 & 1'30" & 35 \\
\hline
\end{tabular}


Table S1.A.4 PCR conditions used for mitochondrial genes

\begin{tabular}{|c|c|c|c|c|c|c|}
\hline \multirow[t]{9}{*}{ Thaumetopoea pinivora } & FW primer & RV primer & MIX & $\mathrm{AT}\left({ }^{\circ} \mathrm{C}\right)$ & EXT & $n^{\circ}$ cycles \\
\hline & C1J2183 & TKN3772 & 1 & 52 & $2^{\prime}$ & 35 \\
\hline & C1J2441 & TKN3785 & 1 & 50 & $2^{\prime}$ & 35 \\
\hline & C1J2441 & C2N3661 & 1 & 52 & $1^{\prime} 30^{\prime \prime}$ & 35 \\
\hline & allC2J3505 & A6N4552m & 1 & 55 & $1^{\prime} 30^{\prime \prime}$ & 35 \\
\hline & A6J4463 & COIIIrev & 1 & 55 & 1'15" & 35 \\
\hline & allco1for & allco3rev & 1 & 61 & 2'30" & 40 \\
\hline & C3if & C3ir & 1 & 55 & $1^{\prime}$ & 35 \\
\hline & TpinA6C3for & TpinA6C3rev & 1 & 50 & $1^{\prime}$ & 35 \\
\hline \multirow[t]{30}{*}{ Theumetopoea pityocampa } & FW primer & RV primer & MIX & AT $\left({ }^{\circ} \mathbf{C}\right)$ & EXT & $n^{\circ}$ cycles \\
\hline & TFJ6400 & N5N7793 & 1 & 48 & 2'30" & 35 \\
\hline & PRND51R & N4N8727 & 1 & 56 & 2'30" & 30 \\
\hline & N5J7077 & N4N8727 & 1 & 51 & $1^{\prime}$ & 35 \\
\hline & N5J7806 & N4N8727 & 1 & 52 & 1'30" & 35 \\
\hline & ochlNAD5for & N4N8727 & 1 & 48 & $45^{\prime \prime}$ & 35 \\
\hline & CBJ10933 & N1N12595 & 1 & 52 & $2^{\prime}$ & 35 \\
\hline & CBJ11335 & N1N12442 & 1 & 52 & $1^{\prime}$ & 35 \\
\hline & CBJ11545 & LRN12866 & 2 & 49 & $1^{\prime}$ & 35 \\
\hline & N1J12261m & LRN13000 & 1 & 62 & $2^{\prime}$ & 35 \\
\hline & ochlcobF1 & LRN12866 & 1 & 56 & $1^{\prime} 45^{\prime \prime}$ & 35 \\
\hline & ochlNAD1for & ochl16Srev & 1 & 55 & $1^{\prime}$ & 35 \\
\hline & N1J12261m & SRN14220 & 2 & 50 & $15^{\prime \prime}$ & 40 \\
\hline & N1J12585m & SRN14220 & 1 & 52 & $2^{\prime}$ & 35 \\
\hline & LRJ12888 & SRN14220 & 1 & 55 & $1 ' 30 "$ & 35 \\
\hline & LRJ13342 & SRN14745 & 1 & 56 & $2^{\prime}$ & 35 \\
\hline & SRJ14197 & TMN200 & 1 & 56 & $1^{\prime} 45^{\prime \prime}$ & 35 \\
\hline & LCO1490 & HCO2198 & 1 & 56 & 2'30" & 30 \\
\hline & C1J1718 & TL2N3014 & 1 & 53 & $2^{\prime}$ & 35 \\
\hline & C1J1718 & C1N2353 & 1 & 56 & $1^{\prime} 45^{\prime \prime}$ & 35 \\
\hline & C1J1751 & C2N3661 & 1 & 53 & $2^{\prime}$ & 35 \\
\hline & allC1J1922 & allC1N3120 & 1 & 53 & $2^{\prime}$ & 35 \\
\hline & C1J2183 & C1N2659 & 1 & 61 & 1'30" & 35 \\
\hline & C1J2441 & C2N3661 & 1 & 54 & 1'30" & 35 \\
\hline & C1J2441 & TKN3785 & 1 & 57 & $2^{\prime}$ & 35 \\
\hline & C2J3399 & A8N4061 & 1 & 55 & $1^{\prime}$ & 35 \\
\hline & allC2J3505 & A8N4061 & 1 & 50 & $1^{\prime}$ & 30 \\
\hline & TKJ3790 & Tpina6c3rev & 1 & 54 & 2'30" & 35 \\
\hline & C3if & C3ir & 1 & 56 & 2'30" & 30 \\
\hline & TKJ3790 & C3N4908 & 1 & 58 & $45^{\prime \prime}$ & 35 \\
\hline
\end{tabular}


Table S1.A.5 PCR conditions used for mitochondrial genes

\begin{tabular}{|c|c|c|c|c|c|c|}
\hline \multirow[t]{27}{*}{ Thaumetopoea pityocampa ENA } & FW primer & RV primer & MIX & AT $\left({ }^{\circ} \mathrm{C}\right)$ & EXT & $n^{\circ}$ cycles \\
\hline & ttun6600 & ttun7806 & 1 & 54 & 1'15" & 35 \\
\hline & allnd5form & MPE_a_nad5 & 1 & 50 & $1^{\prime} 45^{\prime \prime}$ & 35 \\
\hline & allnd5form & allnd4rev & 1 & 55 & 1'30" & 35 \\
\hline & N5J7806 & N4N8727 & 1 & 55 & $2^{\prime}$ & 35 \\
\hline & CBJ10612 & CBN11010 & 1 & 58 & 1'30" & 35 \\
\hline & tuncobforbis & tuncobrevbis & 1 & 54 & $1^{\prime}$ & 35 \\
\hline & CBJ11335 & allnd1rev & 1 & 55 & 1'30" & 35 \\
\hline & CBJ11545 & N1N12595 & 1 & 53 & 1'30" & 35 \\
\hline & CBJ11485 & allnd1rev & 1 & 55 & 1'30" & 35 \\
\hline & ochl16S12Sfor & SRN14220 & 1 & 61 & 1'30" & 35 \\
\hline & LRJ12887 & LRN13398 & 1 & 58 & $1^{\prime}$ & 35 \\
\hline & ttun13206 & ttun13740 & 2 & 60 & $1^{\prime}$ & 35 \\
\hline & LRJ13342 & SRN14220 & 1 & 60 & $1^{\prime}$ & 35 \\
\hline & LRJ13900 & SRN14588 & 1 & 60 & $1^{\prime}$ & 35 \\
\hline & LRJ13900 & SRN14220 & 1 & 60 & $1^{\prime}$ & 35 \\
\hline & pther1612for & pther1612rev & 1 & 54 & 1'30" & 35 \\
\hline & SRJ14197 & SRN14745 & 1 & 54 & 1'30" & 35 \\
\hline & SRJ14197 & TMN200 & 1 & 61 & $2^{\prime}$ & 35 \\
\hline & SRJ14610 & TMN200 & 1 & 60 & $1^{\prime}$ & 35 \\
\hline & LCO1490 & HCO2198 & 1 & 50 & $1^{\prime}$ & 35 \\
\hline & C1J1718 & C1N2353 & 1 & 50 & $1^{\prime}$ & 35 \\
\hline & C1J1718 & TL2N3014 & 1 & 55 & 1'30" & 35 \\
\hline & allC1J1922 & allC1N3120 & 1 & 52 & 1'30" & 35 \\
\hline & C1J2792 & TKN3796 & 1 & 49 & 1'30" & 40 \\
\hline & TKJ3790 & C3N4908 & 1 & 54 & 1'30" & 35 \\
\hline & ttun3900 & $\operatorname{ttun} 4540$ & 2 & 60 & $1^{\prime}$ & 35 \\
\hline \multirow[t]{14}{*}{ Thaumetopoea processionea } & FW primer & RV primer & MIX & $\mathrm{AT}\left({ }^{\circ} \mathrm{C}\right)$ & EXT & $\mathrm{n}^{\circ}$ cycles \\
\hline & TFJ6400 & trnand5rev & 1 & 53 & $2^{\prime}$ & 35 \\
\hline & PRND51R & N4N8727 & 1 & 54 & 2'30" & 35 \\
\hline & N5J7077 & N4N8727 & 1 & 51 & $1^{\prime} 45^{\prime \prime}$ & 35 \\
\hline & allnd5form & MPE_a_nad5 & 1 & 50 & $1^{\prime} 45^{\prime \prime}$ & 35 \\
\hline & N5J7572 & N4N8727 & 1 & 53 & $1^{\prime} 45^{\prime \prime}$ & 35 \\
\hline & pf7925 & pr8776 & 1 & 55 & 1'30" & 35 \\
\hline & CBJ10612 & CBN11010 & 1 & 58 & 1' $30^{\prime \prime}$ & 35 \\
\hline & CBJ10933 & TS1N11683 & 1 & 52 & $1^{\prime}$ & 30 \\
\hline & tpea_0710nd5f & tpea_0710nd5r & 1 & 55 & $1^{\prime}$ & 35 \\
\hline & CBJ11335 & N1N12442 & 1 & 55 & 1'30" & 35 \\
\hline & tpea_0710cob2f & tpea_0710cob2r & 1 & 55 & $1^{\prime}$ & 35 \\
\hline & N1J12261m & LRN12866 & 1 & 61 & $2^{\prime}$ & 35 \\
\hline & N1J12261m & LRN13000 & 2 & 52 & $2^{\prime}$ & 35 \\
\hline
\end{tabular}


Table S1.A.6 PCR conditions used for mitochondrial genes

\begin{tabular}{|c|c|c|c|c|c|c|}
\hline \multirow[t]{12}{*}{ Thaumetopoea processionea } & FW primer & RV primer & MIX & $\mathbf{A T}\left({ }^{\circ} \mathbf{C}\right)$ & EXT & $\mathrm{n}^{\circ}$ cycles \\
\hline & LRJ12887 & LRN13398 & 1 & 52 & $1^{\prime}$ & 30 \\
\hline & LRJ12888 & 12Srev2 & 1 & 60 & 1'45" & 33 \\
\hline & 16Sfor2 & 12Srev2 & 1 & 50 & 1' $30 "$ & 35 \\
\hline & SRJ14197 & TMN200 & 1 & 61 & $2^{\prime}$ & 35 \\
\hline & SRJ14233 & SRN14588 & 1 & 52 & $1^{\prime}$ & 30 \\
\hline & LCO1490 & HCO2198 & 1 & 52 & $1^{\prime}$ & 30 \\
\hline & allC1J1922 & allC1N3120 & 1 & 52 & 1'30" & 35 \\
\hline & C1J2183 & TKN3772 & 1 & 52 & $2^{\prime}$ & 35 \\
\hline & C1J2441 & TKN3785 & 1 & 48 & $2^{\prime}$ & 35 \\
\hline & C2J3399 & A8N4061 & 1 & 52 & $1^{\prime}$ & 35 \\
\hline & TKJ3790 & C3N4908 & 1 & 62 & $2^{\prime}$ & 35 \\
\hline \multirow[t]{23}{*}{ Thaumetopoea solitaria } & FW primer & RV primer & MIX & AT $\left({ }^{\circ} \mathrm{C}\right)$ & EXT & $n^{\circ}$ cycles \\
\hline & TFJ6400 & MPE_a_nad5 & 1 & 55 & 1'30" & 35 \\
\hline & allnd5form & MPE_a_nad5 & 1 & 50 & 1'45" & 35 \\
\hline & pf7925 & pr8776 & 1 & 55 & 1'30" & 35 \\
\hline & N5J7572 & N4N8727 & 1 & 53 & 1'45" & 35 \\
\hline & N53f & N4N8727 & 1 & 50 & 1'30" & 35 \\
\hline & CBJ11485 & N1N12442 & 1 & 50 & 1'30" & 35 \\
\hline & OC7rr & allnd1rev & 1 & 54 & 1'30" & 35 \\
\hline & nd1micro & eochl7rev & 1 & 55 & $45^{\prime \prime}$ & 35 \\
\hline & N1J12261m & LRN13000 & 2 & 52 & $1^{\prime}$ & 35 \\
\hline & LRJ12888 & LRN13398 & 1 & 57 & $1^{\prime}$ & 35 \\
\hline & LRJ12888 & OC10rr & 1 & 61 & 1'30" & 35 \\
\hline & eochl9for & OC10rr & 1 & 54 & 1'15" & 35 \\
\hline & tsol16Sfor & tsol16Srev & 1 & 58 & $1^{\prime}$ & 35 \\
\hline & LCO1490 & HCO2198 & 1 & 50 & $1^{\prime}$ & 35 \\
\hline & SRJ14197 & TMN200 & 2 & 61 & 1'30" & 35 \\
\hline & C1J1751 & C1N2659 & 1 & 52 & 1'30" & 35 \\
\hline & allC1J1922 & allC1N3120 & 1 & 52 & 1'30" & 35 \\
\hline & C1J2441 & TKN3785 & 1 & 50 & $2^{\prime}$ & 35 \\
\hline & C1J2441 & C2N3661 & 1 & 52 & 1'30" & 35 \\
\hline & allC2J3505 & allC3N4923 & 2 & 55 & 1'30" & 35 \\
\hline & C3if & C3ir & 1 & 55 & $1^{\prime}$ & 35 \\
\hline & TKJ3790 & C3N4908 & 1 & 62 & $2^{\prime}$ & 35 \\
\hline \multirow[t]{4}{*}{ Thaumetopoea wilkinsoni } & FW primer & RV primer & MIX & AT $\left({ }^{\circ} \mathbf{C}\right)$ & EXT & $\mathrm{n}^{\circ}$ cycles \\
\hline & PRND51R & N4N8727 & 1 & 54 & 2'30" & 35 \\
\hline & allnd5form & MPE_a_nad5 & 1 & 50 & $1^{\prime} 45^{\prime \prime}$ & 35 \\
\hline & nad5hwF & nad5hwR & 1 & 55 & $1^{\prime}$ & 35 \\
\hline
\end{tabular}


Table S1.A.7 PCR conditions used for mitochondrial genes

\begin{tabular}{|c|c|c|c|c|c|c|}
\hline \multirow[t]{23}{*}{ Thaumetopoea wilkinsoni } & FW primer & RV primer & MIX & $\mathrm{AT}\left({ }^{\circ} \mathrm{C}\right)$ & EXT & $n^{\circ}$ cycles \\
\hline & N53f & N4N8727 & 1 & 50 & 1'30" & 35 \\
\hline & N5J7572 & N4N8727 & 1 & 53 & 1'45" & 35 \\
\hline & twilki0710nd5f & twilki0710nd5r & 1 & 52 & $1^{\prime}$ & 35 \\
\hline & CBJ10933 & allnd1rev & 2 & 57 & 1'30" & 35 \\
\hline & CBJ11335 & allnd1rev & 1 & 55 & 1'30" & 35 \\
\hline & OC7rr & allnd1rev & 1 & 54 & 1'30" & 35 \\
\hline & CBJ11545 & LRN12866 & 1 & 57 & 1'30" & 35 \\
\hline & N1J12261m & LRN13000 & 2 & 52 & $1^{\prime}$ & 35 \\
\hline & twilki0710cobf & twilki0710cobr & 1 & 52 & $1^{\prime}$ & 35 \\
\hline & LRJ12888 & LRN13398 & 1 & 57 & $1^{\prime}$ & 35 \\
\hline & ochl16S12Sfor & OC10rr & 1 & 62 & 1'15" & 35 \\
\hline & SRJ14197 & TMN200 & 1 & 61 & $2^{\prime}$ & 35 \\
\hline & LCO1490 & HCO2198 & 1 & 50 & $1^{\prime}$ & 35 \\
\hline & C1J1718 & TL2N3014 & 1 & 48 & 1'30" & 35 \\
\hline & allC1J1922 & allC1N3120 & 1 & 52 & 1'30" & 35 \\
\hline & C1J2441 & TKN3785 & 1 & 50 & $2^{\prime}$ & 35 \\
\hline & $\mathrm{C} 1 \mathrm{~J} 2441$ & C2N3661 & 1 & 52 & 1'30" & 35 \\
\hline & twilki0710cox1f & twilki0710cox1r & 1 & 52 & $45^{\prime \prime}$ & 35 \\
\hline & C1J2441 & TKN3785 & 1 & 50 & $2^{\prime}$ & 35 \\
\hline & eochl14for & eochl14rev & 1 & 54 & $2 ' 30$ & 35 \\
\hline & C2J3399 & A8N4061 & 1 & 48 & $1^{\prime}$ & 35 \\
\hline & TKJ3790 & C3N4908 & 1 & 62 & $2^{\prime}$ & 35 \\
\hline
\end{tabular}


Table S1.A.8 PCR conditions used for mitochondrial genes

\begin{tabular}{|c|c|c|c|}
\hline FW primer & 5'-sequence-3' & RV primer & $5^{\prime}$-sequence-3' \\
\hline 071105for 1 & ACTTTTGTATAACCGCAACT & 071105rev2 & AATAAGCACAATAGTTTTTGA \\
\hline 16Sfor2 & GCAGCCCTTCAATCAA & 071105rev5 & TAGTACGAAAGGAATGAATA \\
\hline A6J4463 & TTTATTCATATWATWCCNCAAGG & 12Srev2 & TATTGCCCGTCGCTTT \\
\hline allC1J1922 & GGAATTTCHTCHATTTTAGG & A6N4552m & ATGTCCTGCAATTATATTWGC \\
\hline allC2J3505 & TTTTACСMATAAACAAYCAAATTC & A8N4061 & GAAAATAAGTTWGTTATCATTTTCA \\
\hline allco1for & CAGCTGAACATTCYTATAATGA & allC1N3120 & AAAATAATTTGTTCTATTAARGG \\
\hline allnd5form & АТTСТСТТСААССВАААТСААТАY & allC3N4923 & TTTACCTTGAAGDGTTCCTTCTCG \\
\hline C1J1718 & GGAGGATTTGGAAATTGATTAGTTCC & allco3rev & AWAYATCTCGYCATCATTGRTA \\
\hline C1J1751 & GGATCACCTGATATAGCATTCCC & allcobrev & GGRATAGATCGRAGAATTGC \\
\hline C1J2183 & CAACATTTATTTTGATTTTTTGG & allnd1rev & RCARCCTTTTTCWGATGCTATT \\
\hline C1J2441 & CCTACAGGAATTAAAATTTTTAGATGATTAGC & allnd4rev & AATTATTGGGGSTATRTKGGTTC \\
\hline C1J2792 & ATACCTCGACGTTATTCAGA & C1N2353 & GCTCGTGTATCAATATCTATWCC \\
\hline C2for2 & CССАTCAATAGGAGTAAAAG & C1N2659 & GCTAATCCAGTGAATAATGG \\
\hline C2J3399 & TCTATCGGACAYCAATGATAYTG & C1N2776 & GATAATCTGAATATCGWCGNGG \\
\hline C2J3624 & AATCCAGGTCGATTAAAYCA & C2N3661 & CCACAAATTTCTGAACATTGACCA \\
\hline C3if & TATCAAAAGGATTACGATGAGG & C3ir & CGTCAACAAAGTGTCAATATCA \\
\hline CBJ10612 & CСАTCСАAСАTСTCAGCATGATGAAA & C3N4908 & CGTGAAAYATCTCGTCATCATTG \\
\hline CBJ10933 & TATGTACTACCATGAGGACAAATATC & CBN11010 & TATCTACAGCAAATCCСССТCA \\
\hline CBJ11335 & CAYATTCAACCTGAATGATA & CBN11367 & ATTACACСТССТАATTTATTAGGAAT \\
\hline CBJ11485 & TTCAATTTTACССТАTTAAYCA & CBN11526 & TTCTACAGGTCGRGCTCCGATYCA \\
\hline CBJ11545 & ACATGAATTGGAGCTCGACCAGT & COIIIrev & GACCATGAAATCCTGTTGCTAT \\
\hline eochl10for & TCCAATCTTTCATACAAGTCA & eochl14rev & CAGCCTGATATTGGCATT \\
\hline eochl14for & TTCCССTATATTTTCACCAG & eochl7rev & AAAAAGACGAGAAGACCCTA \\
\hline eochl9for & TAAATATTTGCCGAATTCCT & HCO2198 & TAAACTTCAGGGTGACCAAAAAATCA \\
\hline LCO1490 & GGTCAACAAATCATAAAGATATTGG & LRN12866 & ACATGATCTGAGTTCAAACCGG \\
\hline LRJ12887 & CCGGTCTGAACTCAGATCACGT & LRN13000 & TTACCTTAGGGATAACAGCGTAA \\
\hline LRJ12888 & CCGGTTTGAACTCARATCATGTA & LRN13398 & CССТTGTTTATCAAAАAСАT \\
\hline LRJ13342 & CCTTTGTACRGTCAAAATACYGC & MPE_a_nad5 & CCTGTTTCTGCTTTAGTTCA \\
\hline LRJ13900 & TTTAATAAACYCTGATACAMAAG & N1N12442 & AATTTGRGKATTAATTCCTT \\
\hline N1J12261m & TACTTCGTAAGAAATTGTTTGAGC & N1N12595 & GTAGCATTTTTAACTTTATTAGAACG \\
\hline N1J12585m & GGTCCTTTACGAATCTGAATATAACCT & N4N8727 & AAATCTTTRATTGCTTATTCWTC \\
\hline N53f & GCAGAAATACCAGCCATA & N5N7211m & TTAAAGCATTATTGTTTATGTGTGC \\
\hline N5J7077 & TTAAATCTTTWGARTAAAAYCC & N5N7793 & TTGGGTTGRGATGGNYTAGG \\
\hline N5J7572 & AAAGGAATTTGAGCTCTTTTWGT & nad5hwR & TGGTTTGGGRTTRGTTTCTT \\
\hline N5J7806 & GAAACTAAACCCAAACCATCTCA & nad5pbihR & CATGGTTTATGTTCTTCTGG \\
\hline nad5hwF & TAAAAMCCAGCTAAAAAWGG & OC10rr & TTAAATCAAATCAAGATGCAGA \\
\hline nad5pbihF & AAATACACYCССCСAATAAA & ochl16Srev & TAGATACGTGGTTTGTTGATCC \\
\hline nd1micro & ССААСТАAААССССТААAА & pB12Srev & AATTTTGGTGTTAAATAATTC \\
\hline OC7rr & ATGCAACATTAACACGATTT & pr8776 & TTTGTGGKGTCAAAAATATG \\
\hline ochl16S12Sfor & TTTCATCСАAТСТTTCATACAA & pth1612SAr & AGTCTAATCTGCCCMCTGAT \\
\hline ochlcobF1 & TTCСАСССАТАТTTТTССТТTА & pth1612SBr & AAATCAAATCAAGATGCAGA \\
\hline ochlNAD1for & TAАСТСТСТСТСАССТTCAGCA & pther1612rev & TTTTATATTTTAGGGGATAAGC \\
\hline
\end{tabular}


Table S1.A.10 PCR conditions used for mitochondrial genes

\begin{tabular}{|c|c|c|c|}
\hline FW primer & 5'-sequence-3' & RV primer & 5'-sequence-3' \\
\hline ochlNAD5for & AGCAGAAATACCAGCCATAAGT & SRN14220 & ATATGYACAYATTGCCCGTC \\
\hline pB12Sfor & AATAGGGTATCTAATCCTAGTTT & SRN14588 & AAACTAGGATTAGATACCCTATTAT \\
\hline pf7925 & TTGTCTCAAAGTAGATAAAGCAA & SRN14745 & GTGCCAGCAGYYGCGGTTANAC \\
\hline PRND51r & ATGGAATTCCACATAAAGCTAT & tisp0710nd5r & ATGGTTGGTCATGGCTTATG \\
\hline pth1612SAf & AАAАТАAАTCTCACCACAGG & TKN3772 & GAGACCATTACTTGCTTTCAGTCATCT \\
\hline pth1612SBf & AATCTTTCATWCAAGTCAYCAA & TKN3785 & GTTTAAGAGACCAGTACTTG \\
\hline pther1612for & AATCCTTCATTCAAGTCACC & TKN3796 & ACTATAAAATGGTTTAAGAG \\
\hline SRJ14197 & GTACCYCTACTTTGTTACGACTT & TL2N3014 & TCCAATGCACTAATCTGCCATATTA \\
\hline SRJ14233 & AAGAGCGACGGGCGATGTGT & TMN200 & TCCTTTATATTTGAGGTATGARCC \\
\hline SRJ14610 & ATAATAGGGTATCTAATCCTAGT & tpea_0710nd5r & GCTGCAATAACTAAAAGAGC \\
\hline TFJ6400 & TAATATCTTCAATRTYAARCTCT & tpea0710cob2r & AGTTTGAAATGAAATGTTAATCG \\
\hline tisp0710nd5f & CTCATATTTTTGACCCCACA & tpin0710cobr & AAAGTCTAATCTGCCCACTGA \\
\hline TKJ3790 & CATTAGATGACTGAAAGCAAGTA & tpina6c3rev & TCTTAAAATGGCAATAACATAAGA \\
\hline tpea0710cob2f & ATCAGGGGGCAGATTAGACT & trnand5rev & AACTCATGCGATATTTAAAGC \\
\hline tpea0710nd5f & GAAAATAGGAACCACCCTAA & TS1N11683 & TATTTCTTTATTATGTTTTCAAAAC \\
\hline tpin0710cobf & ССААТТААААСССССАААА & tsol16Srev & TTTGAAATGAAATGTTAATCGT \\
\hline tpina6c3for & TСССТTТСААТТТСТTТАССА & $\operatorname{ttun} 13740$ & TTAAAAAGACGAGAAGACCCTA \\
\hline tsol16Sfor & TTACGCTGTTATCCCTAAGGT & ttun4540 & CATAATGGCAGAGAAATTGAGA \\
\hline ttun13206 & ATTAGAGGATCAACCAGCAACT & ttun7806 & TTTTTGGAGTGGGAAATTATTA \\
\hline ttun3900 & TTGCATTACCATCATTACGACT & tuncobrevbis & TTGTCCTGTAATAATGTAAGGTTC \\
\hline ttun6600 & TGATAACCCCAGCACATATAAA & twilki0710cobr & ACAACCTTTTTCAGATGCTA \\
\hline tuncobforbis & AGGAACAGCCTTCATAGGAT & twilki0710cox1r & AGCTCCTAAAATGGAGGAAA \\
\hline twilki0710cobf & AGCCCGACCTGTTGAAGAC & twilki0710nd5r & AGTAAGATTTGTGATGGGAAT \\
\hline twilki0710cox1f & TTGTAACAGCCCATGCTTT & & \\
\hline twilki0710nd5f & AGTAGGAGCAGCCATAGCC & & \\
\hline
\end{tabular}

\section{Buffer 10X}

$\mathrm{MgCl} 225 \mathrm{mM}$

dNTP's $2 \mathrm{mM}$

primer fw $10 \mu M$

primer rw $10 \mu \mathrm{M}$

$\operatorname{Taq}(5 \mathrm{U} / \mu \mathrm{l})$

DNA sample

H2O

TOTAL

$\begin{array}{ll}\text { Mix } 1 & \text { Mix } 2 \\ 4 \mu \mathrm{l} & 4 \mu \mathrm{I} \\ 2 \mu \mathrm{l} & 1,5 \mu \mathrm{I} \\ 1 \mu \mathrm{l} & 1 \mu \mathrm{I} \\ 1 \mu \mathrm{l} & 0,8 \mu \mathrm{l} \\ 1 \mu \mathrm{l} & 0,8 \mu \mathrm{l} \\ 0,2 \mu \mathrm{l} & 0,2 \mu \mathrm{l} \\ 2 \mu \mathrm{l} & 2 \mu \mathrm{l} \\ 8,8 \mu \mathrm{l} & 9,7 \mu \mathrm{l} \\ 20 \mu \mathrm{l} & 20 \mu \mathrm{l}\end{array}$

AT $=$ annealing Temperature in

${ }^{\circ} \mathrm{C}$

EXT $=$ Extension Time 
Table S1.B.1 PCR conditions used for nuclear genes

\begin{tabular}{|c|c|c|c|c|c|c|}
\hline \multirow[t]{4}{*}{ Ochrogaster lunifer } & gene & forward primer & reverse primer & $\operatorname{AT}\left({ }^{\circ} \mathbf{C}\right)$ & EXT & $n^{\circ}$ cycles \\
\hline & $E F$ & cho & EFrcM4 & 61 & 1'15" & 30 \\
\hline & & EF1 & $\mathrm{EF} 4 \mathrm{~b}$ & 51 & $2^{\prime}$ & 35 \\
\hline & wingless & LepWG1 & LepWG2 & 61 & $1^{\prime} 15^{\prime \prime}$ & 30 \\
\hline \multirow[t]{5}{*}{ Thaumetopoea bonjeani } & gene & forward primer & reverse primer & $\operatorname{AT}\left({ }^{\circ} \mathrm{C}\right)$ & EXT & $\mathbf{n}^{\circ}$ cycles \\
\hline & $E F$ & cho & EFrcM4 & 61 & 1'15" & 30 \\
\hline & & starsky & luke & 50 & $1^{\prime}$ & 35 \\
\hline & & tbonEFfor & tbonEFrev & 60 & $1^{\prime}$ & 35 \\
\hline & wingless & LepWG1 & LepWG2 & 62 & 1'15" & 35 \\
\hline \multirow[t]{7}{*}{ Thaumetopoea herculeana } & gene & forward primer & reverse primer & $\operatorname{AT}\left({ }^{\circ} \mathrm{C}\right)$ & EXT & $\mathbf{n}^{\circ}$ cycles \\
\hline & $E F$ & Cho & EFrcM4 & 61 & 1'15" & 35 \\
\hline & & EF3 & EFrcM4 & 53 & 1'30" & 35 \\
\hline & & Efbtshfor & EFrcM4 & 63 & $1^{\prime}$ & 35 \\
\hline & & pEF1for & pEF1rev & 54 & $50 "$ & 35 \\
\hline & & PtbonEFfor & EF4b & 55 & $1^{\prime}$ & 35 \\
\hline & wingless & LepWG1 & LepWG2 & 62 & 1'15" & 35 \\
\hline \multirow[t]{4}{*}{ Thaumetopoea ispartaensis } & gene & forward primer & reverse primer & $\mathbf{A T}\left({ }^{\circ} \mathbf{C}\right)$ & EXT & $\mathrm{n}^{\circ}$ cycles \\
\hline & $E F$ & cho & EFrcM4 & 61 & 1'15" & 30 \\
\hline & & starsky & luke & 50 & $1^{\prime}$ & 35 \\
\hline & wingless & LepWG1 & LepWG2 & 61 & 1'15" & 30 \\
\hline \multirow[t]{4}{*}{ Thaumetopoea libanotica } & gene & forward primer & reverse primer & $\operatorname{AT}\left({ }^{\circ} \mathbf{C}\right)$ & EXT & $n^{\circ}$ cycles \\
\hline & $E F$ & cho & EFrcM4 & 61 & 1'15" & 30 \\
\hline & & Plibpit5EFfor & Plibpit5EFrev & 55 & $1^{\prime}$ & 35 \\
\hline & wingless & LepWG1 & LepWG2 & 61 & 1'15" & 30 \\
\hline \multirow[t]{4}{*}{ Thaumetopoea pinivora } & gene & forward primer & reverse primer & $\operatorname{AT}\left({ }^{\circ} \mathrm{C}\right)$ & EXT & $n^{\circ}$ cycles \\
\hline & $E F$ & cho & EFrcM4 & 61 & 1'15" & 30 \\
\hline & & starsky & luke & 50 & $1^{\prime}$ & 35 \\
\hline & wingless & LepWG1 & LepWG2 & 61 & 1'15" & 30 \\
\hline \multirow[t]{4}{*}{ Thaumetopoea pityocampa } & gene & forward primer & reverse primer & AT $\left({ }^{\circ} \mathrm{C}\right)$ & EXT & $n^{\circ}$ cycles \\
\hline & $\mathrm{EF}$ & cho & EFrcM4 & 61 & 1'15" & 30 \\
\hline & & Plibpit5EFfor & Plibpit5EFrev & 55 & $1^{\prime}$ & 35 \\
\hline & wingless & LepWG1 & LepWG2 & 61 & $1^{\prime} 15^{\prime \prime}$ & 30 \\
\hline \multirow[t]{4}{*}{ Thaumetopoea pityocampa ENA } & gene & forward primer & reverse primer & $\operatorname{AT}\left({ }^{\circ} \mathrm{C}\right)$ & EXT & $\mathrm{n}^{\circ}$ cycles \\
\hline & $E F$ & Cho & EFrcM4 & 61 & 1'15" & 35 \\
\hline & & Plibpit5EFfor & Plibpit5EFrev & 63 & $45^{\prime \prime}$ & 35 \\
\hline & wingless & LepWG1 & LepWG2 & 61 & 1'15" & 30 \\
\hline
\end{tabular}


Table S1.B.2 PCR conditions used for nuclear genes

\begin{tabular}{|c|c|c|c|c|c|c|}
\hline \multirow[t]{5}{*}{ Thaumetopoea processionea } & gene & forward primer & reverse primer & AT $\left({ }^{\circ} \mathbf{C}\right)$ & EXT & $n^{\circ}$ cycles \\
\hline & $E F$ & cho & EFrcM4 & 61 & 1'15" & 30 \\
\hline & & EF1 & EF4b & 51 & $2^{\prime}$ & 35 \\
\hline & & Efbtshfor & EFrcM4 & 63 & $1^{\prime}$ & 35 \\
\hline & wingless & LepWG1 & LepWG2 & 61 & 1'15" & 30 \\
\hline \multirow[t]{5}{*}{ Thaumetopoea solitaria } & gene & forward primer & reverse primer & $\mathbf{A T}\left({ }^{\circ} \mathbf{C}\right)$ & EXT & $\mathrm{n}^{\circ}$ cycles \\
\hline & $E F$ & EF1 & pEF1rev & 53 & 1'30" & 35 \\
\hline & & Plibpit5EFfor & pEF1rev & 58 & $1^{\prime}$ & 35 \\
\hline & & PtbonEFfor & EF4b & 55 & $1^{\prime}$ & 35 \\
\hline & wingless & LepWG1 & LepWG2 & 61 & 1'15" & 30 \\
\hline \multirow[t]{4}{*}{ Thaumetopoea wilkinsoni } & gene & forward primer & reverse primer & $\mathbf{A T}\left({ }^{\circ} \mathbf{C}\right)$ & EXT & $n^{\circ}$ cycles \\
\hline & $E F$ & cho & EFrcM4 & 61 & 1'30" & 30 \\
\hline & & Plibpit5EFfor & Plibpit5EFrev & 55 & $1^{\prime}$ & 35 \\
\hline & wingless & LepWG1 & LepWG2 & 61 & 1'15" & 30 \\
\hline
\end{tabular}




\section{Table S1.B.3 PCR conditions used for nuclear genes}

\begin{tabular}{|c|c|}
\hline \multicolumn{2}{|l|}{ Primer forward } \\
\hline Cho & GTCACCATCATYGACGC \\
\hline EF1 & GGCCACTTGATCTACAAAT \\
\hline EF3 & TCAAGAAAATYGGTTATAAC \\
\hline Efbtshfor & GGAYTCCACTGAGCCCCCWT \\
\hline LepWG1 & GARTGYAARTGYCAYGGYATGTCTGG \\
\hline pEF1for & ACYGGYCACTTGATCTAC \\
\hline Plibpit5EFfor & CGACTCCGGCAAGTCCAC \\
\hline PtbonEFfor & TTGAGGAAATCAAGAAAGAAG \\
\hline starsky & CACATYAACATTGTCGTSATYGG \\
\hline tbonEFfor & CCCTGCTTGCTTTTACACTT \\
\hline \multicolumn{2}{|l|}{ Primer reverse } \\
\hline EFrcM4 & ACAGCVACKGTYTGYCTCATRTC \\
\hline luke & CATRTTGTCKCCGTGCCAKCC \\
\hline EF4b & GAGAGGAGGGAATTCTTG \\
\hline pEF1rev & AACAATAGTACCAGGCTTGAG \\
\hline Plibpit5EFrev & AGCTGCTTTACACCAAGTG \\
\hline tbonEFrev & TGCATCTCCACAGACTTGAC \\
\hline LepWG2 & ACTCGCARCACCARTGGAATGTRCA \\
\hline \multirow[t]{2}{*}{ Mix } & $\mathbf{A T}=$ annealing Temperature in ${ }^{\circ} \mathbf{C}$ \\
\hline & EXT $=$ Extension Time \\
\hline Buffer 10X & $4 \mu \mathrm{l}$ \\
\hline $\mathrm{MgCl} 225 \mathrm{mM}$ & $2 \mu \mathrm{l}$ \\
\hline dNTP's 2mM & $1 \mu \mathrm{l}$ \\
\hline primer fw $10 \mu \mathrm{M}$ & $1 \mu \mathrm{l}$ \\
\hline primer rw $10 \mu \mathrm{M}$ & $1 \mu \mathrm{l}$ \\
\hline $\operatorname{Taq}(5 \mathrm{U} / \mu \mathrm{l})$ & $0,2 \mu \mathrm{l}$ \\
\hline DNA sample & $2 \mu \mathrm{l}$ \\
\hline $\mathrm{H2O}$ & $8,8 \mu \mathrm{l}$ \\
\hline TOTAL & $20 \mu \mathrm{l}$ \\
\hline
\end{tabular}

